\title{
Vers une orientation translittéracique des modèles
} d'information Literacy?

\section{Towards a Transliteral Orientation for Information Literacy \\ Models}

\section{Hacia una orientación transalfabética de los modelos de information literacy}

Karine Aillerie

Volume 61, numéro 4, octobre-décembre 2015

URI : https://id.erudit.org/iderudit/1033434ar

DOI : https://doi.org/10.7202/1033434ar

Aller au sommaire du numéro

Éditeur(s)

Association pour l'avancement des sciences et des techniques de la documentation (ASTED)

ISSN

0315-2340 (imprimé)

2291-8949 (numérique)

Découvrir la revue

Citer cet article

Aillerie, K. (2015). Vers une orientation translittéracique des modèles d'information Literacy?. Documentation et bibliothèques, 61(4), 137-147. https://doi.org/10.7202/1033434ar
Résumé de l'article

Cette contribution vise à interroger les évolutions possibles des modèles " prescriptifs » de la recherche d'information au vu des apports conceptuels de la notion de translittératie au champ de l'information literacy. Y sont abordées les transformations des référentiels de maîtrise de l'information traditionnels ainsi que deux propositions inédites : le modèle américain Metaliteracy et le modèle anglais InFlow (information flow). Ces deux modèles ne se revendiquent pas explicitement de l'approche translittératique même si des points de jonction forts peuvent être repérés qui renouvellent en profondeur les dispositifs de formation à l'information ainsi que l'arrière-plan théorique qui les sous-tend.
Tous droits réservés (C Association pour l'avancement des sciences et des techniques de la documentation (ASTED), 2015
Ce document est protégé par la loi sur le droit d'auteur. L'utilisation des services d’Érudit (y compris la reproduction) est assujettie à sa politique d'utilisation que vous pouvez consulter en ligne. 


\title{
Vers une orientation translittéracique des modèles d'information literacy?
}

\author{
KARINE AiLLERIE \\ Documentaliste certifiée \\ Docteur qualifié en Sciences de l'information et de la communication [Université Paris 13] \\ Chargée d'études [Direction de la recherche et du développement sur les usages du numérique éducatif \\ - Canopé ex Centre National de Documentation Pédagogique] \\ Chercheure associée à l'équipe TECHNE [EA6316 - Université de Poitiers] \\ Membre de l'ANR TRANSLIT ${ }^{1}$
}

\section{RÉSUMÉ | ABSTRACT | RESUMEN}

Cette contribution vise à interroger les évolutions possibles des modèles "prescriptifs" de la recherche d'information au vu des apports conceptuels de la notion de translittératie au champ de l'information literacy. Y sont abordées les transformations des référentiels de maitrise de l'information traditionnels ainsi que deux propositions inédites : le modèle américain Metaliteracy et le modèle anglais InFlow (information flow). Ces deux modèles ne se revendiquent pas explicitement de l'approche translittératique même si des points de jonction forts peuvent être repérés qui renouvellent en profondeur les dispositifs de formation à l'information ainsi que l'arrière-plan théorique qui les sous-tend.

\section{Towards a Transliteral Orientation for Information Literacy Models}

The purpose of this article is to examine the possible evolution of the prescriptive models used for information research in the light of the concepts associated with transliteracy in the field of information literacy. The modifications made to the reference guides used for the mastery of traditional information as well as two unpublished proposals are considered; the American model of metaliteracy and the English model of InFlow (information flow). Both models do not explicitly advocate a transliteral approach even though they share several points in common that renew the information training methods as well as the theory that support them.

\section{Hacia una orientación transalfabética de} los modelos de information literacy

Esta contribución tiene por objetivo analizar las posibles evoluciones de los modelos "prescriptivos" de la búsqueda de información, en función de los aportes conceptuales de la noción de transalfabetización en el campo de la information literacy (alfabetización informacional). Se abordan las modificaciones de los referenciales tradicionales de dominio de la alfabetización informacional, así como dos propuestas inéditas: el modelo estadounidense Metaliteracy (metalfabetización) y el modelo inglés InFlow (information flow; flujo informacional). Estos dos modelos no reivindican explícitamente la postura transalfabética, aunque pueden detectarse puntos de confluencia que renuevan en profundidad los dispositivos de desarrollo de habilidades informativas, así como el fundamento teórico que los respalda.

\section{Introduction}

I notion d'information literacy, traduite en français par les expressions " littératie informationnelle », " maîtrise de l'information " ou «culture(s) de l'information ", porte en elle la question de son opérationnalisation dans des cadres de formation. Ce questionnement est international, mais il renvoie en France à l'article fondateur de Sylvie Chevillote, «Bibliothèques et Information Literacy : un état de l'art ». Cette contribution date de 2005, ce n'est pas si loin, qui déjà pointait la multiplication des évolutions sociotechniques et les difficultés subséquentes à penser et à mettre en pratique des dispositifs efficaces de formation à l'information. Depuis, le paysage médiatique a considérablement changé; problématiques sociales et enjeux fondamentaux, à savoir formalisation des habiletés info-documentaires et mise en œuvre des apprentissages référents, sont plus que jamais d'actualité. Nous ne nous étendrons pas sur la description de la mutation numérique et des usages du Web 2.o, largement définis et commentés par ailleurs. Retenons cependant que les évolutions à prendre en compte se traduisent en premier lieu par la généralisation des interactions directes de l'usager avec les contenus, une " désintermédiation " apparente, revendiquée en tout cas par les outils de recherche grand public et les modèles économiques auxquels ils se rapportent. En second lieu, c'est l'aspect social, réticulaire, de la pratique numérique qui s'est affirmé. Cette sollicitation accrue de l'usager va de pair avec des exigences de formation considérablement renforcées : validation a posteriori de l'information, esprit critique, compétences sociales et collaboration, gestion de la présence numérique. Avec la modification des environnements médiatiques, les usages se transforment, que la recherche travaille à décrire et que les médiateurs (enseignants, bibliothécaires, etc.) travaillent à traduire dans des dispositifs de formation. Le champ de l'information literacy (IL) couvre à la fois ces deux aspects et évolue continûment en fonction des avancées théoriques et des

1. "La translittératie : vers la transformation de la culture de l'information " $<$ http://translit.espe-aquitaine.fr $>$. 
expériences de terrain. Il se structure ainsi autour de la production de modèles, au sein desquels Jérôme Dinet et André Tricot (2008) distinguent les modèles « descriptifs » (modèles cognitifs de la recherche d'information) et les modèles " prescriptifs » (cadres de formation et référentiels de compétences). Nous concentrerons notre attention sur ces derniers.

Au tournant des années 2000, l'approche translittératique, que nous définirons plus avant, s'est agrégée aux débats en cours sur l'IL, s'adjoignant ainsi une dimension formative. Nous posons ici la question d'une évolution possible des modèles de formation à l'information : assiste-t-on à une évolution translittératique de ces modèles prescriptifs? Pour apporter des éléments de réponse à cette question, nous souhaitons aborder dans cet article deux initiatives anglo-saxonnes, dont la première est plutôt orientée vers l'enseignement supérieur et la seconde, vers l'enseignement scolaire, primaire et secondaire : le projet Metaliteracy et le modèle InFlow. Mais avant de regarder de plus près ces deux propositions, nous reviendrons sur le caractère évolutif du concept d'information literacy et aborderons les relations entre IL et translittératie. Nous définirons ensuite ce qu'il est possible d'entendre par modèles de la recherche d'information et exposerons les différentes critiques qui leur sont adressées, les modèles Metaliteracy et InFlow se positionnant comme deux réponses possibles à ces remises en cause.

\section{Information literacy : un concept vivant}

Héritier d'une triple origine à la fois organisationnelle, bibliothéconomique et citoyenne (Le Deuff 2008), l'information literacy (IL) est un sujet majoritairement investi par le monde des bibliothèques, représenté au niveau international par un nombre conséquent de publications scientifiques ou d'associations et de ressources en ligne (p. ex. <infolit.org $>$, <projectinfolit. org $>$, <informationliteracy.org.uk>). C'est aussi une notion riche d'amendements ou de discussions récurrents, Spiranec et Zorica (2010) utilisant l'expression " information literacy movement " pour désigner ce flot conceptuel. Limberg, Sundin et Talja (2012) proposent un pluriel systématique ("information literacies ») pour souligner la multiplicité des acceptions de l'expression suivant les perspectives théoriques et les contextes auxquels elle se rapporte. Même limitée au champ de la francophonie, la littérature qui propose différentes acceptions de la notion d'IL est abondante. La définition bibliothéconomique classique de l'IL se rapporte à celle proposée par l'American Library Association : " être compétent dans l'usage de l'information signifie que l'on sait reconnaître quand émerge un besoin d'information et que l'on est capable de trouver l'information adéquate, de l'évaluer et de l'exploiter" (ALA 1989 cité par Bernhard 1998). Depuis lors, cette définition a dépassé le cadre de l'enseignement universitaire et des biblio- thèques pour s'inscrire au cœur de la formation et de l'apprentissage tout au long de la vie : «Elle permet aux gens, dans tous les chemins de la vie, de chercher, d'évaluer, d'utiliser et de créer l'information pour des objectifs personnels, sociaux, professionnels et éducationnels. C'est un droit humain de base dans un monde numérique qui apporte l'intégration de tous les peuples. » (UNESCO 2005) En France, l'enjeu n'a pas été uniquement de trouver une traduction correcte pour cette expression de langue anglaise, mais surtout de définir les valeurs et les compétences qu'elle implique et par-là même de structurer des dispositifs de formation adéquats. L'expression «maîtrise de l'information » est usuellement employée pour parler en français d'information literacy. Elle fait cependant débat et lui ont été opposées les notions plus complexes de «culture informationnelle " ou de "culture(s) de l'information ", qui lui reprochent le parti pris idéologique de l'adaptation à la société dite " de l'information » et à ses exigences économiques, ainsi qu'une approche pédagogique trop procédurale et décontextualisée (Serres 2009).

Ainsi vit l'IL, au gré de ses conceptualisations, se nourrissant des évolutions sociotechniques auxquelles elle est relative. Les nouvelles acceptions de l'IL ou ses contextualisations nationales n'effacent pas en soi les définitions précédentes mais viennent les enrichir. Les années 2000 ont cependant vu proliférer toute une série de littératies voisines de l'IL, voire candidates à sa relégation : media literacy, digital literacy, cyberliteracy, visual literacy, mobile literacy, ICT literacy, computer literacy, library literacy, information fluency, etc. Nous souhaiterions nous arrêter un moment sur une forme bien particulière de littératie, parfois présentée elle aussi comme émergente, mais qui présente toutefois un caractère radicalement spécifique : la «translittératie " (transliteracy). Ce concept est en effet susceptible de subsumer une bonne partie de ces littératies multiples - celles-ci risquant parfois de ne faire sens qu'associées à une technologie ou à un domaine de compétence particulier - et de redonner ainsi une nouvelle dimension à l'IL. Il s'agit pour les auteurs qui le défendent de bien plus qu'une littératie parmi d'autres, cette notion présentant un caractère fédérateur, à la hauteur des enjeux sociotechniques du moment et pouvant permettre de rompre avec des approches disciplinaires par trop étanches (Delamotte, Liquète \& Frau-Meigs 2014).

\section{De l'information literacy à la « translittératie »}

La translittératie est issue de travaux universitaires anglo-saxons en communication et en cultural studies. Ils étudient les aspects techniques, sociaux et cultu- 
rels des nouveaux médias et de la lecture numérique ${ }^{2}$, et s'interrogent sur les rapports entre numérique, écriture et littérature ${ }^{3}$. Alan Liu recourt au terme " translittératies » pour appréhender une expérience de lecture au sens large, "remixant des compétences de lecture anciennes et nouvelles" $(2012,5)$. Il parle dès lors de "reconfiguration", la lecture demeurant le même geste d'un point de vue anthropologique, mais apparaissant transformé à l'aune des relations entre anciens et nouveaux supports de lecture, entre anciennes et nouvelles façons de lire. Étymologiquement, la translittératie fait appel à la "translittération », qui désigne le passage d'un système d'écriture à un autre, la transcription littérale d'un alphabet à un autre et inversement (p. ex. la norme ISO 233-2 : 1993 pour la transcription des caractères arabes). Insistant sur ce caractère "translatif ", la définition la plus citée de la translittératie est aujourd'hui celle proposée par Sue Thomas et ses collègues : "Transliteracy is the ability to read, write and interact across a range of platforms, tools and media from signing and orality through handwriting, print, $T V$, radio and film, to digital social networks. » $(2007,1)$

L'approche translittératique ne provient donc pas directement du champ des sciences de l'information et des bibliothèques, mais elle prolonge et élargit la notion d'IL. Malgré une certaine frilosité de départ, le monde des bibliothèques tend en partie à la faire sienne. Citons ici par exemple le blogue <librariesandtransliteracy.wordpress.com>, animé par la bibliothécaire et chercheuse Bobbi Newman. Lancé afin de disséminer la notion de translittératie en bibliothèque, ce blogue a été fermé en 2012, la mission étant considérée comme accomplie par ses concepteurs. La translittératie fait effectivement sens face au concept d'IL et à sa nature évolutive, car elle permet de souligner la nécessité de travailler non pas tant à des alphabétisations relatives à des supports spécifiques qu'à un niveau d'expertise globale qui prend en compte les interactions entre toutes ces littératies (Ipri 2010). Notons que ce caractère essentiellement transversal et transdisciplinaire était déjà en germe dans les travaux fondateurs sur l'IL. En effet, Paulette Bernhard (2001), analysant au début des années 2000 différents référentiels de formation à l'information, insistait sur la prétention de tous à la transposition des compétences d'une situation à l'autre.

Le champ de compétences lié à l'IL ou maîtrise de l'information s'est aujourd'hui complexifié. Prenons comme exemple la validation de l'information, qui relève aujourd'hui essentiellement de la seule responsabilité de l'usager : elle doit pouvoir s'exercer à la fois dans un catalogue de bibliothèque, face aux résultats d'un moteur de recherche grand public, sur un réseau

2. Voir le projet «Transliteracies » coordonnée par Alan Liu, Université de Californie à Santa Barbara, 2005-2010. <http://transliteracies.english.ucsb.edu/category/ research-project>.

3. Voir le projet "Production and Research in Transliteracy ", coordonné par Sue Thomas, Université de Montfort à Leicester, 2006-2013. social ouvert de type Facebook, tout comme dans le contexte d'une interaction directe ou instrumentée entre plusieurs personnes. Le relais entre IL et translittératie impose pourtant à cette dernière d'endosser des préoccupations de formation qu'elle n'avait pas initialement. Delamotte et al. $(2014,11)$ propose ainsi de définir la translittératie à la lumière des cultures de l'information comme "l'ensemble des compétences d'interaction mises en ouvre par les usagers sur tous les moyens d'information et de communication disponibles: oral, textuel, iconique, numérique,... essentiellement dans des environnements et contextes numériques ». Ces interactions translittératiques renvoient à des habiletés de recherche et de traitement de l'information : savoir "chercher, évaluer, valider, modifier, stocker, organiser, diffuser l'information selon ses contextes d'usage (le code, l'actualité, le document...) plus ou moins explicites et formalisés " (Delamotte et al. 2014, 12). Il y va de capacités d'anticipation du fonctionnement et du potentiel informationnel des dispositifs communicationnels, de compétences métacognitives et d'organisation (p. ex. suivi d'un projet mené en contexte scolaire et en dehors, répartition des tâches dans le cadre d'un travail collectif), de savoir-faire procéduraux adaptés à un environnement donné (individus, contexte, technologie, situation), et d'un accent mis sur les modes de communication de l'information recueillie puis traitée. Ces modes de communication, qui modifient profondément le statut de la production académique et de son évaluation, dépassent largement la relation duale entre un enseignant commanditaire et un étudiant/élève exécutant pour s'inscrire dans une situation de communication réelle, reposant sur une organisation collective et souvent réalisée hors l'espace-temps de formation.

Englobant plusieurs domaines de pratique (informationnelle, médiatique, informatique), rejoignant l'idée de "technologies intellectuelles" (Robert 2000) et l'ancrage ethnologique de la notion de littératie (Hoggart 1957, Goody 1977), la translittératie désigne la transversalité des habiletés informationnelles (d'un support ou d'un genre éditorial à l'autre) et l'enchevêtrement sociotechnique des contextes (scolaires, professionnels, de loisir, publics, privés, etc.). Si la notion originale, basée sur les pratiques quotidiennes ("Everyday life in a transliterate world (Thomas et al. 2007), n'inclut pas au départ de visées proprement pédagogiques, en France, elle rassemble et confronte les éducations à l'information, aux médias et à l'informatique et invite la notion d'information literacy à un enrichissement conceptuel vers les cultures de l'information. Elle implique un dépassement des postures pédagogiques vers la prise en compte de la globalité et de la complexité des pratiques sociales, scolaires et professionnelles (Delamotte et al. 2014). Pour tendre vers ces objectifs d'éducation de l'usager, la translittératie n'est pas pour le moment directement associée à un "modèle prescriptif » susceptible de l'incarner. L'IL, par contre, 
nous l'avons évoqué, est inséparable de la formalisation des compétences et de la structuration de dispositifs de formation. Étant donné l'audience actuelle de la translittératie, les modèles existants auraient-ils alors assimilé des éléments translittératiques? Avant de poser cette question, revenons toutefois sur cette notion protéiforme de « modèle ».

\section{Modèles de la recherche d'information}

\section{Modèles cognitifs de la recherche d'information}

La modélisation de l'activité de recherche d'information concerne tout un ensemble de recherches théoriques, largement issues de la psychologie cognitive, qui visent à représenter formellement les comportements humains en situation de recherche d'information (RI) et d'interaction avec des systèmes d'information. Ces modèles ont été développés en réaction aux approches technocentrées afin d'approcher la réalité des processus cognitifs de la RI. L'arrivée des systèmes hypertextuels et interconnectés a relancé les tentatives de modélisation du comportement cognitif et manipulatoire de l'individu chercheur d'information. Depuis le modèle en cinq étapes proposé par John Guthrie (1988), basé sur la localisation et l'extraction de l'information, plusieurs modèles de RI ont vu le jour et coexistent, tentant chacun de rendre compte de la nature complexe de cette activité humaine. Citons entre autres le modèle exploratoire dit Berrypicking de Marcia Bates (1989), qui tend à rendre compte du chemin, plus ou moins hasardeux, emprunté par la personne au fur et à mesure de la collecte d'information. Citons aussi le modèle ISP (Information Search Process) en six étapes de Carol Collier Kuhlthau (1993), qui intègre les motivations affectives (incertitude et anxiété) influençant la RI. En France, Jean-François Rouet et André Tricot (1998) élaborent également, sur la base d'une vision cyclique de la RI, le modèle EST (Évaluation, Sélection, Traitement), qui met au jour la coexistence de deux strates cognitives : le processus de base, automatique, et l'optimisation métacognitive, qui visent à élaborer, de par la trajectoire de la recherche, la représentation mentale du but. Dans le prolongement de ces courants de recherche, des auteurs ont pointé l'importance du contexte sociotechnique dans lequel s'élaborent ces pratiques informationnelles (Ihadjadène \& Chaudiron 2009). L'ensemble de ces modèles est qualifié par Dinet et Tricot de "modèles explicatifs ", et ce, par comparaison avec une autre forme de modélisation dite " prescriptive » ("prescriptive guidelines" (Johnston \& Webber 2003)), qui ne vise pas seulement à décrire l'activité de RI, mais à en formaliser l'expertise, base de référentiels utiles aux formateurs (Dinet \& Tricot 2008). Ainsi, lorsqu'il est question de "modèles ", il faut distinguer d'une part ces modélisations théoriques du comportement et d'autre part leurs déclinaisons sous forme de référentiels nationaux et transnationaux. Ce sont plus précisément les modèles dits « prescriptifs » qui nous intéressent ici.

\section{Modèles « prescriptifs » et référentiels pour la maîtrise de l'information}

Les modèles «prescriptifs » de la RI consignent des compétences info-documentaires précises, déclinées à partir des étapes cruciales du processus de RI mises au jour par les modèles explicatifs cités précédemment. L'objectif d'une telle formalisation est d'intégrer ces éléments aux apprentissages disciplinaires et interdisciplinaires de l'enseignement scolaire et universitaire, et de pouvoir les adapter en fonction des spécificités de terrain. Ces modèles peuvent être qualifiés de « référentiels » pour la maîtrise de l'information par les élèves, les étudiants, voire les adultes en formation. Selon Pascal Duplessis (2005, 180), un référentiel renvoie «à une activité concrète et ponctuelle, en l'occurrence une activité de recherche d'information", et est "constitué d'objectifs prescrivant des comportements attendus lors de l'activité considérée ». La synthèse effectuée par Paulette Bernhard entre 2001 et 2005 signale l'existence d'un nombre considérable de ces référentiels développés au sein de différents pays, voire de différents systèmes éducatifs. Ces référentiels sont, dans les grandes lignes, comparables. Citons par exemple le référentiel développé en France par la Fédération des enseignants documentalistes de l'Éducation nationale (FADBEN 1997). Adossées aux écrits de Carol Kuhlthau, les compétences info-documentaires y apparaissent énoncées sous la forme de sept grandes étapes successives (mise en projet, questionnement, repérage, récupération de données, lecture/ écriture, production/communication, évaluation). Ce référentiel s'inscrit en outre dans le contexte précis de la production finale que l'élève doit remettre à l'enseignant prescripteur à l'issu de la recherche effectuée. Il est possible de relever quelques-uns des modèles génériques ayant inspiré ce type de déclinaisons locales :

- Le référentiel Information Literacy Standards for Student Learning publié en 1998 par l'American Association of School Librarians (AASL, ÉtatsUnis), puis élargi et mis à jour en 2007 par la brochure Standards for the 21st-Century Learners, mettant en valeur des activités pédagogiques basées sur la recherche, la collaboration et la production de connaissances par les élèves;

- Le modèle, parmi les plus connus, Big6 Skills de Eisenberg et Berkowitz (États-Unis, traduit en 18 langues, 1999-2003). Ce modèle, d'inspiration comportementale, s'adresse principalement à l'enseignement élémentaire et secondaire. Il détaille 6 étapes (définition de la tâche, stratégies de recherche d'information, localisation et accès, utiliser l'information, synthétiser, évaluer la production et la démarche) qui ensemble défendent une 
approche procédurale et linéaire de la recherche d'information;

- Le comité IL de la Society of College, National and University Libraries (SCONUL, Royaume-Uni et Irlande) a publié en 1999 le référentiel Information Skills in Higher Education: A SCONUL Position Paper, révisé en 2011 sous la forme de sept piliers (Identify, Scope, Plan, Gather, Evaluate, Manage, Present). Ce modèle est organisé autour de 7 domaines de compétences. Chacune de ces compétences se trouve elle-même déclinée en connaissances à acquérir et en comportements à mettre en œuvre en fonction de la situation d'apprentissage et du public visé. La version révisée de ce référentiel tend à rompre avec les représentations linéaires du processus de RI;

- L'Association of College \& Research Libraries (ACRL, États-Unis) a publié un référentiel (ALA 200o) traduit en français sous le titre : Norme sur les compétences informationnelles dans l'enseignement supérieur (CREPUQ 2005) et régulièrement complétée par des programmes de mise en œuvre concrète. Cette approche curriculaire vise à homogénéiser les objectifs d'apprentissage et les exigences dans les différents niveaux de l'enseignement jusqu'à l'université ainsi qu'à réconcilier enseignements disciplinaires et apprentissages info-documentaires. Ce document intègre une dimension collective dans l'utilisation de l'information collectée (utiliser efficacement l'information, individuellement ou en groupe);

- Le Council of Australian University Librarians (CAUL) a édité son référentiel pour l'Australie et la Nouvelle-Zélande en 2001, puis en 2004: New Zealand Information Literacy Framework: Principles, Standards and Practice, reprenant le déroulé méthodologique de la recherche d'information déclinée sous la forme de grandes étapes complémentaires, depuis l'identification du besoin d'information jusqu'à l'utilisation éthique et raisonnée des informations collectées;

- Le Chartered Institute of Information and Library Professionals (CILIP, Royaume-Uni) a publié un référentiel en 2004, reprenant les mêmes compétences. Ce travail vise pour sa part à élargir l'acception de la notion d'IL et des compétences qu'elle recouvre à d'autres domaines que simplement l'éducation ou les bibliothèques, impliquant les professionnels de l'information au sens large, les organismes publics ou gouvernementaux mais aussi le secteur privé, économique et commercial. Adossé en 2011 à la Proclamation d'Alexandrie, ce référentiel a ainsi été élargi aux contextes de la vie quotidienne et professionnelle.

\section{Critiques adressées aux modèles de formation à la maîtrise de l'information}

Ces modèles prescriptifs de la RI, en grande partie conçus avant le déploiement des supports numériques et d'Internet, ont été, et sont encore, largement convoqués dans la littérature et sur le terrain, qu'il s'agisse de la formation des étudiants universitaires ou des élèves de l'enseignement primaire et secondaire. Certains auteurs plaident pourtant pour une refonte de ces standards et, plus profondément, pour une conception renouvelée de l'IL qui en constitue l'arrière-plan théorique : représentation d'un usager passif récipiendaire, consommateur de contenus, et d'une information objet statique ayant vocation à être retrouvé plus que construit. Ils s'attachent ainsi à rendre compatible une "IL 2.0 " avec des contextes infomédiatiques et pédagogiques en pleine évolution (mondes immersifs, réseaux sociaux, sms, podcasts, documents collaboratifs, etc.) et avec les usages réels des technologies (Tuominen 2007; Kutner \& Armstrong 2012; Karsenti et al. 2014). En effet, l'enjeu de formation majeur, pointé depuis quelques années déjà, c'est la capacité effective de l'usager connecté à participer (Jenkins 2006), à produire et à faire (Colegrove 2013). Or, cet usager et les compétences qu'il développe, ou non, dans divers contextes semble échapper de plus en plus aux cadres formels et aux ambitions pédagogiques des professionnels, documentalistes, bibliothécaires, enseignants... Cet usager qui, précisément, est sensé interagir directement avec les outils d'accès et de production de l'information au moyen de terminaux mobiles, de plus en plus puissants et "profilés ". Ses recherches d'information et ses productions scolaires tendent à se dérouler en dehors de l'espace-temps académique et des sphères de médiation, pour se faire à la maison, en ligne, via les réseaux sociaux, etc. Ses pratiques quotidiennes entrent ainsi souvent en dissonance avec l'expertise professionnelle et ses représentations (Cordier 2011).

Concernant les modèles prescriptifs, c'est en premier lieu la réduction des processus cognitifs à une liste de comportements observables et évaluables ainsi que la ponctualité des séances de formation qui ont été très tôt critiquées (Johnston \& Webber 2003; Duplessis 2005). C'est conséquemment la linéarité de ces référentiels et la conformité à de grandes étapes consécutives qui sont remises en cause car inadaptées aux habitudes de navigation sur le Web (Branch \& Oberg 2003; Markless \& Streatfield 2007; Bawden \& Robinson 2009). C'est aussi la dimension sociale et collective des pratiques informationnelles qui est fréquemment absente des modèles existants (Farkas 2012), établis sur la description d'une interaction usager-système essentiellement individuelle. C'est, plus globalement, toute la chaîne du processus informationnel qui serait à repenser, en particulier les modes de production/recréation, de communication, de caractérisation et de partage de l'information; l'organisation des environnements informationnels personnels; 
les modes de recherche et de validation de l'information dans les environnements sociaux. La validation des sources et de l'information prend ici en effet une importance accrue, réévaluée par rapport à la phase de recherche proprement dite privilégiée dans les modèles traditionnels (Serres 2012). À ce titre, certains chercheurs insistent pour repositionner ces compétences de validation en amont et tout au long du processus de recherche d'information, et non pas en dernière partie de processus, une fois l'information collectée, comme décrit dans les référentiels méthodologiques traditionnels, un champ de recherche à part entière se constituant ainsi autour de la critical information literacy (Smith 2013).

L'activité cognitive de la RI dans le contexte du Web 2.0 demeure certes encore largement méconnue (Hyldegård 2009; Boubée \& Tricot 2010). Mais une vision globale, non pas relative seulement à tel ou tel média et à ses codes éditoriaux plus ou moins stabilisés, mais qui corresponde à la réalité des usages tels qu'ils se diffusent dans les sociétés, est nécessaire. La perspective translittératique peut ici permettre de garder à l'esprit quelques principes à la fois simples et déterminants : on ne recherche pas de l'information uniquement sur Internet; les autres supports n'ont pas disparu et ne vont pas disparaître, mais ils vont continuer d'évoluer; sur le Web, nous n'avons pas à faire à un contexte de lecture identique, mais chaque fois à reconstruire. Notons dès lors que les "modèles descriptifs" ne semblent pas encore avoir pris toute la mesure de l'évolution des processus cognitifs et des pratiques sociales dans les environnements actuels, ouverts et collaboratifs. Il faudrait pourtant les adapter et les inscrire dans des dispositifs de formation qui font sens pour les apprenants comme pour les enseignants. Concernant les enseignants français par exemple, les enquêtes donnent à voir un transfert difficile de pratiques numériques personnelles riches vers des pratiques en classe très ponctuelles et utilitaristes (Ministère de l'éducation nationale 2014). Concernant les jeunes, passée l'euphorie du mythe des digital natives, les nombreuses études réalisées à ce jour sur leurs usages numériques conduisent à un consensus quant à l'hétérogénéité de leurs dispositions et des bénéfices retirés (Livingstone \& Helsper 2007; Hatlevik \& Christopherson 2013; Aillerie 2013; Boyd 2014), qui réaffirme crûment la nécessité d'une formation solide pour tous. Ce sont donc les modèles « prescriptifs » qui tendent à se renouveler, prenant de cours en quelque sorte les modélisations théoriques. Nous choisissons d'examiner ici quelques propositions récentes de ces modèles «prescriptifs » intégrant l'aspect collaboratif, « multicontextes » et «multimédias » de l'activité informationnelle.

\section{Vers une transition translittératique des modèles prescriptifs?}

\section{La rénovation des modèles existants}

Récemment, les grands modèles prescriptifs que nous avons listés plus haut, internationaux mais d'inspiration surtout anglo-saxonne, ont été en grande partie amendés. La plupart de ces révisions consistent à sortir le référentiel du seul contexte académique et à multiplier les situations d'application des compétences (lifelong learning). De même, les révisions intègrent différents paliers de formation, de novice à expert. D'un point de vue formel, il est question de casser le déroulé linéaire des référentiels classiques (SCONUL 2011). Il s'agit globalement de réaffirmer l'enjeu politique et d'inclusion sociale de la maîtrise de l'information (empowerment). D'un point de vue conceptuel, ces modèles tendent largement à s'ouvrir aux littératies liées aux dernières technologies, dont nous avons parlé (digital literacy, etc). Mentionnons, à titre d'exemple, le référentiel SCONUL, mis à jour en 2011 : « [I]t was felt that the model needed to be updated and expanded to reflect more clearly the range of different terminologies and concepts which we now understand as "Information Literacy" " (2). Dans le même temps, le champ de l'IL rejoint des problématiques transversales quant aux mutations de la médiation éducative et des processus d'apprentissage : «Learning is enhanced by opportunities to share and learn with others. Students need to develop skills in sharing knowledge and learning with others, both in face-to-face situations and through technology. " (AASL 2007, 3) Finalement, il s'agit plutôt de changer la perspective sur les standards que les standards eux-mêmes, comme dans le cas du référentiel de l'ACRL (2015) modifié en 20132014, non pas tant du point de vue des compétences elles-mêmes que des prérequis théoriques : "Authority Is Constructed and Contextual, Information Creation as a Process, Information Has Value, Research as Inquiry, Scholarship Is a Conversation, Searching Is Strategic. »

Si elles interrogent les mêmes changements sociotechniques, la rénovation des grands modèles de formation à la maîtrise de l'information n'aborde pas la question translittératique en tant que telle. Par ailleurs, la focalisation y est forte sur les technologies connectées. À côté de ces modèles traditionnels et de leurs mises à jour, certains auteurs défendent la possibilité d'élaborer de nouveaux modèles, à partir d'une IL repensée et susceptible de rassembler la multiplicité des littératies émergentes. C'est en premier lieu par leur présentation formelle que ces initiatives se remarquent (schémas, déploiement transmédiatique). Nous proposons de nous arrêter sur deux exemples aboutis et concrètement mis en œuvre ou expérimentés. 


\section{Le projet Metaliteracy de la State University of New York (SUNY)}

Thomas P. Mackey, spécialiste de l'apprentissage à distance, et Trudi E. Jacobson, bibliothécaire à l'Université d'Albany (New York), sont à l'origine du projet Metaliteracy. Ils visent à répondre aux exigences d'un environnement médiatique transformé et de reconsidérer à la fois le concept d'IL, mais aussi, voire surtout, les modalités de la formation à la maîtrise de l'information offerte à l'université. Ils proposent ainsi un nouveau modèle prescriptif, basé sur une définition classique de l'IL ("determine, access, locate, understand and use information " (ACRL 200o), qu'ils repensent à la lumière des médias sociaux et des communautés en ligne. Ils lui ajoutent ainsi les compétences suivantes : " collaborate, produce, participate and share » (Mackey Jacobson 2014, voir figure 1).

Quatre champs d'expertise principaux se trouvent ainsi déclinés qui subsument ces compétences :

- Évaluer les contenus de façon critique, incluant les contenus dynamiques, c'est-à-dire des contenus en ligne qui se modifient et évoluent constamment comme les prépublications scientifiques, les blogs ou les wikis;

- Comprendre les enjeux éthiques reliés à l'information, à la protection des données personnelles et à la propriété intellectuelle dans des environnements technologiques mouvants;

- Partager l'information et collaborer au sein de divers environnements participatifs;

- Connecter les apprentissages et les stratégies de recherche avec les projets de formation tout au long de la vie, les desseins personnels, scolaires et professionnels.

(Mackey \& Jacobson 2014)

\section{Figure 1}

Modèle métalittératique (Mackey \& Jacobson 2014, 23)

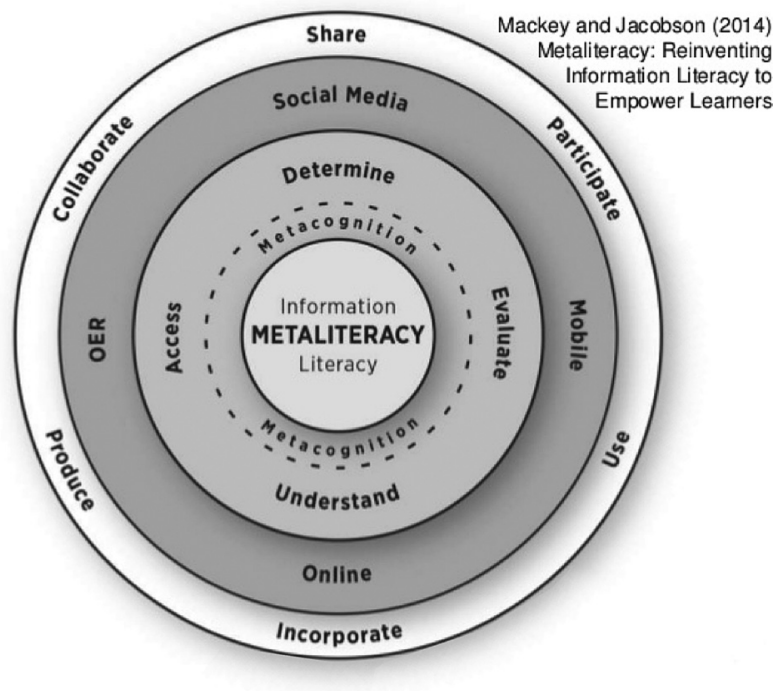

Comme le montre la figure 1, les compétences " en entrée ", centrées sur la détermination du besoin d'information et sur l'accès aux sources, répondent à d'autres « en sortie » axées sur l'activité de production d'informations nouvelles et sur la participation (modification, partage) de l'usager. En termes de ressources, et comme le montre toujours la figure 1, l'accent est mis sur les ressources à caractère collectif ou collaboratif (Open Educational Resources, médias sociaux), accessibles et modifiables quel que soit le contexte (mobilité). Le projet Metaliteracy accorde en outre une place centrale aux habiletés métacognitives dans l'activité informationnelle, et c'est d'ailleurs en ce sens qu'est utilisé le préfixe « méta ».

Ce préfixe positionne également le modèle Metaliteracy vis-à-vis des modèles existants et des littératies émergentes. D’abord déployé sous la forme d'un article de recherche (Mackey \& Jacobson 2011), d'un site Internet $(<$ metaliteracy.learningtimes.net $>)$, d'un blog $(<$ metaliteracy.org $>$ ), d'un MOOC ( $<$ metaliteracy.cdlprojects. com>), puis d'un livre (Mackey \& Jacobson 2014), s'accompagnant d'études de cas et de maquettes de formation, ce projet propose une approche globale, capable d'unifier les multiples visages de l'IL (" expanded, overarching and integrated framework ", Mackey \& Jacobson 2014,19$)$. Il prévoit de mobiliser les disciplines universitaires autour de champs de compétences jusqu'alors implicites et non intégrés aux référentiels existants : la production, la collaboration, le partage de l'information et la métacognition. Il vise ainsi à adosser les apprentissages disciplinaires à la participation active, à la production et au partage de l'information, brute ou remixée, au sein des environnements collaboratifs. L'objectif est de recentrer l'effort pédagogique des outils vers les pratiques réelles et les besoins des étudiants. La formation ellemême y est pensée de manière collaborative, entre enseignants, bibliothécaires, experts et apprenants.

Selon Dunaway (2011), la métalittératie et la translittératie témoignent d'une approche commune d'inspiration connectiviste. L'ouvrage de Mackey et Jacobson (2014) confronte à plusieurs reprises « métalittératie » et " translittératie », et souligne en effet le point commun essentiel entre ces deux approches, à savoir rassembler les différentes littératies, traditionnelles et émergentes. Cependant, l'IL constitue la base de la métalittératie qui revendique son appartenance première au champ classique des sciences de l'information et des bibliothèques, ce qui n'est pas le cas, nous l'avons dit, de la translittératie. Mackey et Jacobson se distinguent également de l'approche translittératique, car celle-ci ne propose pas à ce jour d'objectifs de formation précis ${ }^{4}$. La translittératie est ainsi traitée par Mackey et Jacobson (2014) au même rang que les littératies émergentes que la métalittératie vise à agréger.

4. C'est en partie l'objet du projet ANR TRANSLIT («La translittératie : vers la transformation de la culture de l'information ») 2012-2016 <http://translit.espe-aquitaine.fr $>$. 


\section{Du projet iTEC (Innovative Technologies for an Engaging Classroom) au modèle InFlow}

Le modèle InFlow (Information Flow) a été élaboré au sein de l'Education and Social Research Institute (ESRI) de l'Université de Manchester (MacNicol 2014). Il s'inscrit dans les résultats en cours de publication d'un projet de recherche-action européen intitulé iTEC (innovative Technologies for an Engaging Classroom), coordonné par l'organisme European Schoolnet et financé dans le cadre du $7^{\mathrm{e}}$ programme-cadre de la Commission européenne pour la recherche et le développement technologique. Mis en œuvre dans 18 pays entre 2010 et 2014, il visait à tester des scénarios pédagogiques en classe, scénarios inspirés de la pensée créatrice (design thinking) impliquant l'usage de technologies numériques ainsi que la mise en œuvre par les élèves du CE2 à la Terminale de compétences de type inquiry based learning (collecte de données, recherche d'information, collaboration, communication, métacognition). Partant de l'idée que les habiletés informationnelles sont implicitement présentes dans nombre de séances pédagogiques, le modèle InFlow vise à abandonner les approches isolant des compétences pour proposer des situations pédagogiques au cours desquelles les apprenants sont amenés à interagir de façon explicite avec l'information suivant un ensemble d'activités variées. Comme le montre la figure 2, celles-ci se présentent sous la forme de huit modules centrés sur la génération et la mobilisation d'idées (imagine, ask), la collecte de données (explore), la production de ressources par les élèves (make), incluant la conception de projet et sa communication (map, show). Occupent ici également une place fondamentale les activités métacognitives (reflect) ainsi que la coopération ou la collaboration (collaborate) entre élèves, entre élèves et enseignants, voire entre enseignants (Figure 2). Ces modules peuvent être mis en ouvre de manière non successive, voire itérative. Ce modèle est actuellement expérimenté en milieu scolaire au Royaume-Uni.

\section{Figure 2}

InFlow (Information Flow):

An Integrated Model of Applied Information Literacy. Model Summary

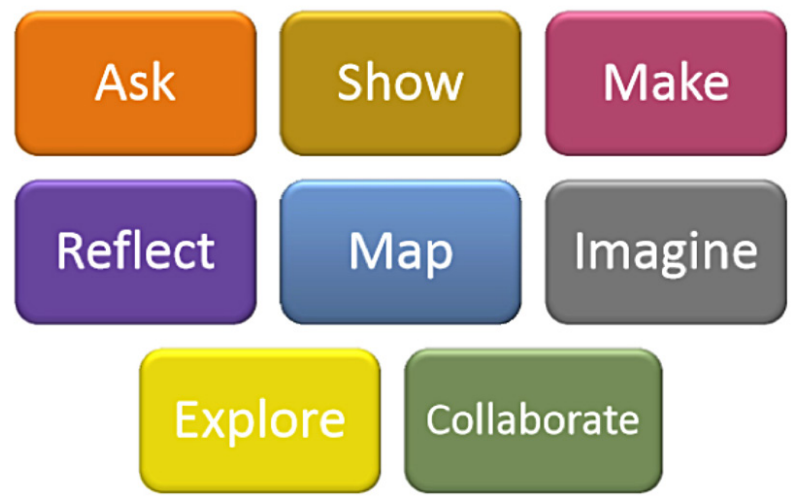

Le modèle InFlow ne se réfère pas explicitement à la translittératie. Il se situe néanmoins dans le prolongement d'une vision critique de l'IL et vise une meilleure adéquation avec les compétences du XXI siècle (Binkley et al. 2012), se rapprochant ainsi de la vision " $i$-skills " proposée par The Joint Information Services Committee (JISC) : "The ability to identify, assess, retrieve, evaluate, adapt, organise and communicate information within an iterative context of review and reflection." (JISC 2005) En ce sens, il vise à combler l'écart qu'il regrette entre les modèles prescriptifs de l'IL et les "pédagogies 2.0 ». La recherche d'information y est d'autre part définie de manière étendue, c'est-à-dire au-delà des environnements éditoriaux traditionnels, intégrant la collecte de données brutes, la recherche d'information dans les réseaux sociaux, l'interrogation directe de personnes. Il milite également pour une prise en compte forte des processus de création, de révision, de réécriture, etc. Ce sont deux points de jonction qu'il nous semble possible d'établir entre la translittératie et le modèle InFlow.

\section{Des modèles translittératiques?}

En proposant une approche résolument non linéaire et tâchant de prendre en compte à la fois les parcours individuels et les dynamiques collectives, les initiatives Metaliteracy et InFlow marquent une rupture avec les référentiels traditionnels de formation à l'information, eux-mêmes en évolution. Par ailleurs, beaucoup de ces référentiels sont structurés autour de différents paliers d'expertise, en porte-à-faux par rapport aux pratiques actuelles. Les modèles Metaliteracy et InFlow mettent en avant la coconstruction des pratiques, non seulement entre élèves ou étudiants, mais également entre élèves et professeurs, entre novices et experts, entre amateurs et professionnels. Il ne s'agit pas tant de mettre à niveau les compétences effectives de chacun que d'intégrer dans le projet d'enseignement et d'apprentissage les pratiques réelles plutôt que supposées des uns et des autres.

Toutefois, ni le modèle métalittératique ni le modèle InFlow ne se revendiquent de l'approche translittératique. Ils partagent néanmoins avec la translittératie des points de rencontre qu'il nous semble crucial d'envisager. En premier lieu, ils tentent d'apporter une réponse au défi d'intégrer les compétences numériques, effectivement souvent décrites en dehors du champ de l'IL, tout en souhaitant bénéficier de la richesse des réflexions menées à propos du concept d'IL et en particulier les plus récentes sur ses évolutions nécessaires. Ils rejoignent ainsi chacun la notion de translittératie dans la délimitation d'un noyau de compétences répondant à la difficulté d'appréhension d'environnements sociotechniques mouvants et par l'affirmation d'une forme de "métacompétence " apte à embrasser la multitude des contextes infomédiatiques (" crossing divides » selon 
Sue et al. 2007). Ils choisissent d'autre part de mettre de l'avant des domaines de compétences relevant au mieux de l'implicite dans les modèles de formation classiques : évaluation critique de l'information sous les formats les plus divers, repérage et construction de l'information envisagés de manière collaborative, partage de l'information et modalités afférentes. Enfin, en insistant sur les processus de production et de création de l'information et des savoirs, sur leur complexité et sur leur niveau d'exigence, ces initiatives révèlent une ambition de formation à la hauteur de l'usager "translettré ». Si ces deux modèles ne se revendiquent pas explicitement de la translittératie, ils se rejoignent sur le constat commun suivant lequel les modèles IL doivent évoluer pour prendre en compte la multiplicité des supports et des contextes d'interaction avec les médias, la dimension collaborative et de partage de l'information, la capacité à produire de l'information. Il y va d'un réajustement entre littératies du lire et écrire et compétences nouvelles. Ces deux initiatives prolongent aussi l'approche translittératique en proposant des situations de formation concrètes, adaptables et transférables. Elles interrogent ainsi profondément le travail scolaire ou académique et l'engagement de l'apprenant dans ces activités.

\section{Conclusion}

Les modèles prescriptifs de la RI sont aujourd'hui rattrapés par l'urgence de revoir la formation à la mâ̂trise de l'information, face à la remise en cause des médiations traditionnelles et à la généralisation des pratiques numériques individuelles. Des initiatives prennent forme alors qu'une distorsion est constatée entre pratiques sociales et standards de l'information literacy, les compétences déclinées par ces référentiels ne correspondant plus totalement aux modes actuels d'accès et de traitement de l'information. Ils devancent en cela, pour ainsi dire, les modèles théoriques descriptifs qui les ont au départ inspirés. Sont ainsi remis au goût du jour les référentiels traditionnels et apparaissent des modèles revendiquant une nouveauté plus ou moins radicale par rapport à leurs prédécesseurs : les modèles Metaliteracy et InFlow en sont deux exemples. L'irruption de la notion de translittératie dans le champ des sciences de l'information et des bibliothèques représente une étape importante dans le parcours conceptuel de l'information literacy. Il est à noter toutefois que cette notion n'a pas été reprise en tant que telle par les modèles prescriptifs rénovés ou récents. Cela dit, des éléments fondateurs de la translittératie y sont bels et bien présents, à savoir : situer la littératie informationnelle dans tous les supports de communication à disposition, de l'imprimé au numérique, incluant l'audiovisuel, les interactions directes, etc.; sortir la littératie informationnelle du seul contexte académique formel; rompre avec une perspective binaire et contre-productive du numérique opposé à l'imprimé. Cependant, la rénovation des modèles classiques tend à se focaliser sur les technologies numériques, ne faisant pas forcément le lien de manière explicite avec les supports imprimés et audiovisuels classiques et n'insistant pas sur le caractère possiblement transférable des compétences, voire sur l'identification d'une compétence à part entière liée à la flexibilité. Les deux modèles originaux que sont Metaliteracy et InFlow visent à rassembler les différents médias et littératies dans une perspective globale telle que se propose de la conceptualiser l'approche translittératique. L'usager n'y est plus seulement l'élève ou l'étudiant, par opposition au professionnel, car ces modèles se fondent sur un décentrement du formateur vers les potentialités individuelles et collectives des usagers. Ces modèles, eux-mêmes conçus comme évolutifs et collaboratifs, ne s'adressent plus seulement aux professionnels de l'information mais aux médiateurs en général et vise à engager activement les apprenants dans leur rapport à l'information et au savoir. Du point de vue des institutions éducatives, il s'agit de repenser le rôle de l'enseignant en général dans l'élaboration et la coconstruction des connaissances. À la base de ces modèles, nous retrouvons l'objectif d'explicitation de compétences cruciales et l'affirmation de la nécessité incontestable de former l'usager dont les pratiques sont très diverses et les profils très hétérogènes. Il s'agit bien là de donner aux individus la possibilité d'incarner un usager participant et «translettré ». À ce titre, de telles initiatives, parmi d'autres encore à venir, ont le mérite de provoquer la réflexion. Elles doivent cependant être éprouvées sur le terrain, épreuve en cours pour les deux modèles. Du point de vue de la recherche, le pendant inévitable de ces expérimentations in situ consiste à affiner notre connaissance des pratiques réelles, formelles et informelles, des usagers, élèves, étudiants et formateurs. Ce sont ces deux facettes inséparables l'une de l'autre qui contribuera à l'élaboration de cadres de formation pertinents et compatibles avec les pratiques sociales.

\section{Sources consultées}

Association of College and Research Libraries (ACRL). 2015. Framework for Information Literacy for Higher Education. $<$ www.ala.org/acrl/standards/ilframework> (consulté le 8 juillet 2015).

Advisory Committee on Information Literacy. 1999. Information Skills in Higher Education: A SCONUL Position Paper. <www. sconul.ac.uk/sites/default/files/documents/Seven_pillars2.pdf $>$ (consulté le 8 mars 2015).

Aillerie, Karine. 2013. Engagement personnel et prescription scolaire dans les usages informationnels de l'internet. In Culturenum: politiques culturelles et éducatives dans la vague numérique, sous la direction de Hervé Le Crosnier. Caen : C\&F Éditions, 51-71.

American Association of School Librarians (AASL). 2007. Standards for the 21st-Century Learners. Chicago: American Association of School Librarians 
< http://www.ala.org/aasl/standards-guidelines/learningstandardshttp://www.ala.org/aasl/sites/ala.org.aasl/files/ content/guidelinesandstandards/learningstandards/AASL_ LearningStandards.pdf $>$ (consulté le 25 août 2015).

American Association of School Librarians (AASL). 1998. Information Literacy Standards for Student Learning: Standards and indicators. American Library Association, Association for Educational Communications and Technology

$<$ http://umanitoba.ca/libraries/units/education/media/ InformationLiteracyStandards_final.pdf> (consulté le 25 août 2015).

American Library Association (ALA). 1989. Presidential Committee on Information Literacy: Final Report. Chicago : American Library Association.

Bates, Marcia. 1989. The design of browsing and berrypicking techniques for the online search interface. Online Review 13 (5) : 407-424.

Bawden, David \& Lyn Robinson. 2009. The darkside of information: Overload, anxiety and other paradoxes and pathologies. Journal of Information Science 35 (2) : 180-191.

Bernhard, Paulette. 1998. Apprendre à « maîtriser » l'information: des habiletés indispensables dans une " société du savoir ». Éducation et francophonie 26 (1). <http://www.acelf.ca/c/ revue/revuehtml/26-1/o9-bernhard.html> (consulté le 24 août 2015).

Bernhard, Paulette. 2001. Examen d'un ensemble de modèles de processus de recherche d'information et proposition de modélisation <mapageweb.umontreal.ca/bernh/TICI/modeles.html> (consulté le 8 mars 2015).

Binkley, Marilyn, Ola Erstad, Joan Herman, Senta Raizen, Martin Ripley, May Miller-Ricci \& Mike Rumble. 2012. Defining twenty-first century skills. In Assessment and Teaching of $21^{\text {st }}$ Century Skills, sous la direction de Patrick Griffin, Barry McGaw \& Esther Care. Dordrecht : Springer Netherlands, $17-66$.

Boubée, Nicole \& André Tricot. 2010. Qu'est-ce que rechercher de l'information? Villeurbanne: Presses de l'enssib.

Boyd, Danah. 2014. It's Complicated: The Social Lives of Networked Teens. New Haven : Yale University Press.

Branch, Jennifer \& Dianne Oberg. 2003. The British models. School Library Media Activities Monthly 19 (10) : 17-24.

Bundy, Alan (dir.). 2004. Australian and New Zealand Information Literacy Framework: Principles, Standards and Practice. Adelaide : Australian and New Zealand Institute for Information Literacy. <www.caul.edu.au/content/upload/files/ info-literacy/InfoLiteracyFramework.pdf> (consulté le 8 mars 2015).

Chevillote, Sylvie. 2005. Bibliothèques et Information Literacy: un état de l'art. Bulletin des bibliothèques de France 50 (2) : 42-49.

Colegrove, Patrick. 2013. Editorial board thoughts: Libraries as makerspace? Information Technology and Libraries 32 (1) : 2-5.

Conférence des recteurs et des principaux des universités du Québec (CREPUQ). 2005. Norme sur les compétences informationnelles dans l'enseignement supérieur de l'Association of College \& Research Libraries (ACRL). <www.crepuq.qc.ca/ IMG/pdf/normeacrl-web-03-05-v4.pdf> (consulté le 8 juillet 2015).

Cordier, Anne. 2011. Formaliser l'activité de recherche d'information : les modèles et modélisations à l'épreuve des pratiques effectives. Colloque international du réseau MUSSI (réseau franco-brésilien), Toulouse. <sic_00642953> (consulté le 8 mars 2015).

Delamotte, Eric, Vincent Liquète \& Divina Frau-Meigs. 2014. La translittératie, à la convergence des cultures de l'information : supports, contexte et modalités. Spirale (53) : 145-156.
Dinet, Jérôme \& André Tricot. 2008. Recherche d'information dans les documents électroniques. In Ergonomie des documents électroniques, sous la direction d'Aline Chevalier \& André Tricot. Paris : Presses universitaires de France, 35-69.

Dunaway, Michelle Kathleen. 2011. Connectivism: Learning theory and pedagogical practice for networked information landscapes. Reference Services Review 39 (4) : 675-685.

Duplessis, Pascal. 2005. L'enjeu des référentiels de compétences infodocumentaires dans l'Éducation nationale. DocumentalisteSciences de l'information 42 (3) : 178-189.

Fédération des enseignants documentalistes de l'Éducation nationale (FADBEN). 1997. Référentiel : compétences en information-documentation. <www.fadben.asso.fr/IMG/pdf/ REFERENTIEL-COMPETENCES-1997-2.pdf> (consulté le 8 mars 2015).

Farkas, Meredith. 2011. Information literacy 2.o. American Libraries $42(11-12): 32$.

Goody, Jack. (1977) The Domestication of the Savage Mind. Cambridge University Press

Guthrie, John T. 1988. Locating information in documents: Examination of a cognitive model. Reading Research Quarterly 23 (2) : 178-199.

Ipri, Tom. 2010. Introducing transliteracy: What does it mean to academic libraries? College and Research Libraries News 71 (10) : 532-567.

Karsenti, Thierry, Gabriel Dumouchel \& Vassilis Komis. 2014. Les compétences informationnelles des étudiants à l'heure du Web 2.0 : proposition d'un modèle pour baliser les formations. Documentation et bibliothèques 60 (1) : 20-30.

Hatlevik, Ove Edvard \& Knut-Andreas Christophersen. 2013. Digital competence at the beginning of upper secondary school: Identifying factors explaining digital inclusion. Computers \& Education 63 (avril) : 240-247.

Hoggart, Richard. 1957. The uses of literacy: Aspects of WorkingClass Life (Penguin Modern Classics). Penguin books,

Hyldegård, Jette. 2009. Beyond the search process: Exploring group members' information behavior in context. Information Processing \& Management 45 (1) : 142-158.

Ihadjadene, Madjid \& Stéphane Chaudiron. 2009. Des processus aux pratiques : quels modèles informationnels pour analyser l'accès à l'information en contexte professionnel? In Évolutions technologiques et information professionnelle: pratiques, acteurs et documents. Colloque international organisé par le GRESEC, Université Stendhal, 10 et 11 décembre, 1-12.

Jenkins, Henri. 2006. Convergence Culture: Where Old and New Media Collide. New York : New York University Press.

Johnston, Bill \& Sheila Webber. 2003. Information literacy in higher education: A review and case study. Studies in Higher Education 28 (3) : 335-352.

Joint Information Systems Committee (JISC). 2005. Investing in Staff i-Skills. JISC, 45p.

Kuhlthau, Carol Collier. 1993. Seeking Meaning: A Process Approach to Library and Information Services. Norwood: Ablex Publishing Corp.

Kutner, Laurie \& Alison Armstrong. 2012. Rethinking information literacy in a globalized world. Communications in Information Literacy 6 (1) : 24-33.

Le Deuff, Olivier. 2008. La culture de l'information : Quelles «littératies » pour quelles conceptions de l'information? In $V I^{e}$ Colloque international du chapitre français de l'ISKO, 7 et 8 juin 2007. Toulouse : IUT de l'Université Paul Sabatier, 97-116.

Limberg, Louise, Olof Sundin \& Sanna Talja. 2012. Three theoretical perspectives on information literacy. Human IT: Journal for Information Technology Studies as a Human Science 11 (2): 91-128. 
Liu, Alan. 2012. Translitteraties : le big bang de la lecture en ligne. In E-dossiers de l'audiovisuel : l'éducation aux cultures de l'information, sous la direction de Divina Frau-Meigs, Éric Bruillard \& Éric Delamotte, Eric. <www.ina-expert. com/e-dossier-de-l-audiovisuel-l-education-aux-culturesde-l-information/translitteraties-le-big-bang-de-la-lectureen-ligne.html> (consulté le 8 mars 2015).

Livingstone, Sonia \& Ellen Helsper. 2007. Gradations in digital inclusion: Children, young people and the digital divide. New Media Society 9 (4) : 671-696.

Mackey, Thomas P. \& Trudi E. Jacobson. 2011. Reframing information literacy as a metaliteracy. College \& Research Libraries $72(1): 62-78$.

Mackey, Thomas P. \& Trudi E. Jacobson. 2014. Metaliteracy: Reinventing information literacy to empower learners. Chicago : Neal-Schuman Publishers.

Markless, Sharon \& David Streatfield. 2007. Three decades of information literacy: Redefining the parameters. In Change and Challenge: Information Literacy for the $21^{\text {st }}$ Century, sous la direction de Susie Andretta. Adelaide : Auslib, 15-36.

$\mathrm{McNicol}$, Sarah. 2014. Modelling information literacy for classrooms of the future. Journal of Librarianship and Information Science $1(2): 2-11$.

Ministère de l'éducation nationale (MEN). 2014. Enquête PROFETIC auprès de 5 ooo enseignants du second degré : rapport. <cache.media.eduscol.education.fr/file/ETIC_et_ PROFETIC/58/o/PROFETIC-2014-rapport_346580.pdf> (consulté le 8 mars 2015).

Robert, Pascal. 200o. Qu'est-ce qu'une technologie intellectuelle? Communication et langages (123) : 97-114.

Rouet, Jean-François \& André Tricot. 1998. Chercher de l'information dans un hypertexte. In Les hypermédias, approches cognitives et ergonomiques, sous la direction d'André Tricot \& Jean-François Rouet. Paris : Hermès, 57-74.

Serres, Alexandre. 2009. Penser la culture informationnelle: des difficultés de l'exercice. Les Cahiers du numérique (5) 3:9-23.

Serres, Alexandre. 2012. Dans le labyrinthe : évaluer l'information sur internet. Caen : C\&F Éditions.

Smith, Laureen. 2013. Towards a model of critical information literacy instruction for the development of political agency. Journal of Information Literacy 7 (2) : 15-32.

Spiranec, Sonja \& Mihaela Banek Zorica. 2010. Information literacy 2.0: Hype or discourse refinement? Journal of Documentation (66) $1: 140-153$.

Thomas, Sue, Chris Joseph, Jess Lacetti, Bruce Mason, Simon Mills, Simon Perril \& Kate Pullinger. 2007 (3 décembre). Transliteracy: Crossing divides. First Monday 12 (12). < firstmonday.org/article/view/2060/1908> (consulté le 8 mars 2015).

Tuominen, Kimmo. 2007. Information literacy 2.o. Signum 40 (5) : 6-12.

Unesco, Ifla, Nfil. 2005. La proclamation d'Alexandrie sur la maîtrise de l'information et l'apprentissage tout au long de la vie. Colloque international sur la maîtrise de l'information et la formation tout au long de la vie. Alexandrie, 6-9 novembre. 